\title{
Sufficient conditions for existence of solutions of a lower dimensional variational problem
}

\author{
José Matias \\ Centro de Análise Matemática, Geometria e Sistemas Dinâmicos, \\ Departamento de Matemática, \\ Instituto Superior Técnico \\ Av. Rovisco Pais, 1 \\ 1049-001 Lisboa, Portugal \\ email: jmatias@math.ist.utl.pt
}

\begin{abstract}
Solutions of variational problems under affine boundary conditions can be viewed equivalently as solutions of an implicit differential equation coupled with another equation that relates to quasiaffinity on the quasiconvex envelope of the integrand. In this work we derive conditions on an integrand and on its quasiconvex envelope which are sufficient for existence of solutions of a related lower dimensional variational problem derived through a dimension reduction process.
\end{abstract}

\section{Introduction}

Existence of solutions of the problem

$$
(P) \quad \inf \left\{\int_{\Omega} f(\nabla u(x)) d x, u \in W^{1, \infty}\left(\Omega ; \mathbb{R}^{d}\right) \quad u=u_{\zeta_{0}} \text { on } \partial \Omega\right\}
$$

where $\Omega$ is an open bounded subset of $\mathbb{R}^{N}$ and $f: \mathbb{R}^{d \times N} \rightarrow \mathbb{R}, N, d \geq 1$ with $u_{\zeta_{0}}$ a given affine map, has been studied extensively by many authors. We refer to [17] and [18] and the references therein.

As usual the case where $N=1$ or $d=1$ will be denoted by scalar and the case where $N, d>1$ by vectorial. We recall that in the scalar case quasiconvexity and convexity are equivalent notions.

2000 Mathematics Subject Classification. MATHEMATICS SUBJECT CLASSIFICATION 34A60, 35E10, 49J45, 74K35.

Key words: Calculus of Variations, Dimension Reduction. 
If $f$ is quasiconvex the problem $(P)$ trivially has $u_{\zeta_{0}}$ has a minimizer. As derived in [17], when $f$ fails to be quasiconvex, $(P)$ attains a solution if and only if there exists $\bar{u} \in u_{\zeta_{0}}+W_{0}^{1, \infty}\left(\Omega ; \mathbb{R}^{d}\right)$ such that

$$
f(\nabla \bar{u}(x))=Q f(\nabla \bar{u}(x)) \text { a.e. } x \in \Omega
$$

and

$$
\int_{\Omega} Q f(\nabla \bar{u}(x)) d x=Q f\left(\zeta_{0}\right)|\Omega|
$$

where $Q f$ is the quasiconvex envelope of $f$, namely

$$
Q f=\sup \{g \leq f: g \text { quasiconvex }\} .
$$

Equations (1.1) and (1.2) are of a different nature as pointed out for instance in [14]. Equation (1.1) is what is called an implicit partial differential equation (see [18] for historical and bibliographical comments), while (1.2) is more geometrical in nature since it relates to quasiaffinity on the quasiconvex envelope of $f$.

Departing from sufficient conditions for (1.1) and (1.2) to hold (cf. [14]) we intend to derive conditions on $f$ and on its quasiconvex envelope that ensure existence of solutions of a related lower dimensional problem. The energy density of this lower dimensional problem is determined by a dimension reduction process and relates to $f$ through the following definition:

Definition 1.0.1. Let $f: \mathbb{R}^{d \times N} \rightarrow \mathbb{R}$ be a Borel measurable function, satisfying $f(\zeta) \geq-C, \forall \zeta \in \mathbb{R}^{d \times N}$. Writing $\zeta=\left(\zeta_{\alpha} \mid \zeta_{N}\right)$, where $\zeta_{\alpha} \in$ $\mathbb{R}^{d \times(N-1)}$ and $\zeta_{N} \in \mathbb{R}^{d}$, we define $\bar{f}: \mathbb{R}^{d \times(N-1)} \rightarrow \mathbb{R}$ by:

$$
\bar{f}\left(\zeta_{\alpha}\right):=\inf _{\lambda \in \mathbb{R}^{d}}\left\{f\left(\zeta_{\alpha} \mid \lambda\right)\right\}
$$

Since we will deal both with $f: \mathbb{R}^{d \times N} \rightarrow \mathbb{R}$ and with $\bar{f}: \mathbb{R}^{d \times(N-1)} \rightarrow \mathbb{R}$, we will denote by $C_{N} f, Q_{N} f, R_{N} f$ the convex, quasiconvex and rank one convex envelopes of $f$ and by $C_{N-1} \bar{f}, Q_{N-1} \bar{f}, R_{N-1} \bar{f}$ the corresponding envelopes of $\bar{f}$.

Our goal is to derive conditions on $f$ and on the set

$$
K:=\left\{\zeta \in \mathbb{R}^{d \times N}: Q_{N} f(\zeta)<f(\zeta)\right\},
$$

that are sufficient to ensure existence of solutions of the problem:

where

$$
(\bar{P}) \quad \inf \left\{\int_{w} \bar{f}\left(\nabla v\left(x_{\alpha}\right)\right) d x_{\alpha}, \quad v \in v_{\zeta_{0, \alpha}}+W_{0}^{1, \infty}\left(w ; \mathbb{R}^{d}\right)\right\},
$$

$$
w \subset \mathbb{R}^{N-1}, \quad \zeta_{0, \alpha} \in \bar{K}:=\left\{\zeta_{\alpha}: Q_{N-1} \bar{f}\left(\zeta_{\alpha}\right)<\bar{f}\left(\zeta_{\alpha}\right)\right\}
$$


and $v_{\zeta_{0, \alpha}}$ is a given affine map.

More precisely we want to derive conditions on $f$ and $K$ that are sufficient to ensure that $\bar{f}$ and $\bar{K}$ satisfy the hypothesis of [14] (cf. Corollary 3.0.1) which in turn ensure existence of solutions of $(\bar{P})$.

The outline of the paper is as follows: In Section 2 we introduce the notation used throughout the work; in Section 3 we recall the different notions of convexity and some conditions on $f$ and $K$ that are sufficient for (1.1) and (1.2) to hold. We also introduce in Section 3 the conditions on $f$ and $K$ that will be sufficient to ensure existence of solutions of $(\bar{P})$.

In Section 4 we review briefly the dimension reduction result of Le Dret and Raoult in [22] and Section 5 is devoted to the statement and proof of our results. Loosely speaking, our main result (cf. Section 5, Theorem 5.2.1) states that under appropriate hypothesis on $f$ and $K$ ( cf. Definition 3.0.4 iii) and Definition (3.0.6)), problem $(\bar{P})$ attains solutions as long as the quasiaffinity hypothesis on $Q_{N} f$ along rank-one directions are transmitted to $Q_{N-1} \bar{f}$. This holds to be true if $\zeta_{\alpha} \in \bar{K}$ is such that

$$
Q_{N}^{-} f\left(\zeta_{\alpha}\right)=Q_{N-1} \bar{f}\left(\zeta_{\alpha}\right)
$$

Unfortunately (1.3) is not true in general and for that reason we will need an extra condition (cf. Definition 3.0.5) in the statement of Theorem 5.2.1.

We also discuss some cases where it can be shown that (1.3) holds. One such situation is the scalar case and this allows for a simpler statement of our main theorem when $d=1$ (cf. Theorem 5.2.2) since we can drop condition (3.0.5).

Finally we discuss a few examples in Section 5.2.

\section{Notation}

In this section we introduce the notation used in this work.

Throughout the text $N \geq 2$ and $w \subset \mathbb{R}^{N-1}$ will denote an open bounded set with Lipschitz boundary. For $\epsilon>0$ we set $\left.\Omega_{\epsilon}=w \times\right]-\frac{\epsilon}{2}, \frac{\epsilon}{2}[$ and denote simply by $\Omega$ the subset of $\mathbb{R}^{N}$ corresponding to $\left.\Omega_{1}=w \times\right]-\frac{1}{2}, \frac{1}{2}[=w \times I$. Let $\left.\Gamma_{\epsilon}=\partial w \times\right]-\frac{\epsilon}{2}$, $\frac{\epsilon}{2}$ be the lateral surface of $\Omega_{\epsilon}$ and $\Gamma$ be the lateral surface of $\Omega$.

We write $x \in \mathbb{R}^{N}$ as $x=\left(x_{\alpha} \mid x_{N}\right)$, with $x_{\alpha} \in \mathbb{R}^{N-1}$ and $x_{N} \in \mathbb{R}$.

Similarly, if $\zeta \in \mathbb{R}^{d \times N}$, we write $\zeta=\left(\zeta_{\alpha} \mid \zeta_{N}\right)$, where $\zeta_{\alpha} \in \mathbb{R}^{d \times(N-1)}$ and $\zeta_{N} \in \mathbb{R}^{d}$. We will also use the following notations:

- $Q$ denotes the unit cube of $\mathbb{R}^{N}$ centered at the origin with one side orthogonal to $e_{N}$, the $n^{\text {th }}$ vector of the canonical basis of $\mathbb{R}^{N}$,

- when related to $\mathbb{R}^{N-1}$ we write $Q^{\prime}$ in place of $Q$, 
- $C$ represents a generic constant,

- we denote by $B_{\epsilon}$ the ball in $\mathbb{R}^{N}$ centered at the origin with radius $\epsilon$ and by $B_{\epsilon}^{\prime}$ the ball in $\mathbb{R}^{N-1}$ centered at the origin with radius $\epsilon$,

- we identify $\gamma=\left(\gamma_{\alpha} \mid 0\right) \in \mathbb{R}^{N}$ with $\gamma_{\alpha} \in \mathbb{R}^{N-1}$. Similarly, for $\beta \in \mathbb{R}^{d}$ and $\gamma=\left(\gamma_{\alpha} \mid 0\right) \in \mathbb{R}^{N}$, we write $\beta \otimes \gamma_{\alpha} \in \mathbb{R}^{d \times(N-1)}$ in place of $\beta \otimes\left(\gamma_{\alpha} \mid 0\right) \in \mathbb{R}^{d \times N}$.

\section{Preliminaries}

The purpose of this section is to give a brief overview of the concepts and results that are used in the sequel. All these results are stated without proofs as they can be readily found in the references given below. Moreover, we introduce some new definitions.

3.1. Quasiconvex problems in the calculus of variations. We start by recalling the different notions of convexity used throughout the article.

Definition 3.0.2. $\quad$ (i) A Borel measurable function $f: \mathbb{R}^{d \times N} \rightarrow \mathbb{R}$ is said to be quasiconvex if

$$
\int_{U} f(\zeta+\nabla \phi(x)) d x \geq f(\zeta)|U|
$$

for every bounded domain $U \subset \mathbb{R}^{N}, \zeta \in \mathbb{R}^{d \times N}$, and $\phi \in W_{0}^{1, \infty}\left(U ; \mathbb{R}^{d}\right)$.

(ii) A function $f: \mathbb{R}^{d \times N} \rightarrow \overline{\mathbb{R}}=\mathbb{R} \cup\{+\infty\}$ is said to be rank one convex if

$$
f\left(\theta \zeta_{1}+(1-\theta) \zeta_{2}\right) \leq \theta f\left(\zeta_{1}\right)+(1-\theta) f\left(\zeta_{2}\right)
$$

for every $\zeta_{1}, \zeta_{2} \in \mathbb{R}^{d \times N}$ with $\operatorname{rank}\left\{\zeta_{1}-\zeta_{2}\right\}=1$ and every $\theta \in[0,1]$.

(iii) A Borel measurable function $f: \mathbb{R}^{d \times N} \rightarrow \mathbb{R}$ is said to be quasiaffine (or equivalently rank one affine) if both $f$ and $-f$ are quasiconvex.

(iv) The different envelopes of a given function $f$ are defined as

$$
\begin{gathered}
C f=\sup \{g \leq f: g \text { convex }\}, \\
Q f=\sup \{g \leq f: g \text { quasiconvex }\}, \\
R f=\sup \{g \leq f: g \text { rank one convex }\},
\end{gathered}
$$

and the following implications hold

$$
f \text { convex } \Rightarrow f \text { quasiconvex } \Rightarrow f \text { rank one convex }
$$

which leads to

$$
C f \leq Q f \leq R f \leq f .
$$

We remind the following definitions given in [14]:

Definition 3.0.3. Let $K \subset \mathbb{R}^{d \times N}$ be open and $\lambda \in \mathbb{R}^{d \times N}$. 
i) For $\zeta \in K$ we denote by $L_{K}(\zeta, \lambda)$ the largest segment of the form $[\zeta+t \lambda, \zeta+s \lambda], t<0<s$, so that $(\zeta+t \lambda, \zeta+s \lambda) \subset K$.

ii) If $L_{K}(\zeta, \lambda)$ is bounded, we denote by $t_{-}(\zeta)<0<t_{+}(\zeta)$ the elements so that $L_{K}(\zeta, \lambda)=\left[\zeta+t_{-} \lambda, \zeta+t_{+} \lambda\right]$. They therefore satisfy

$$
\zeta+t_{ \pm} \lambda \in \partial K, \zeta+t \lambda \in K \quad \forall t \in\left(t_{-}, t_{+}\right) .
$$

iii) If $H \subset K$, we let

$$
L_{K}(H, \lambda)=\bigcup_{\zeta \in H} L_{K}(\zeta, \lambda)
$$

In order to address a lower dimensional problem involving $\bar{f}$ we recall some definitions given in [14] and introduce a new one (definition iii) below):

Definition 3.0.4. (Boundedness and stable boundedness in a direction $\lambda$ ). Let $K \subset \mathbb{R}^{d \times N}$ be open, $\zeta_{0} \in K$ and $\lambda \in \mathbb{R}^{d \times N}$.

i) We say that $K$ is bounded at $\zeta_{0}$ in the direction $\lambda$ if $L_{K}\left(\zeta_{0}, \lambda\right)$ is bounded.

ii) We say that $K$ is stably bounded at $\zeta_{0}$ in the rank-one direction $\lambda=\beta \otimes \gamma\left(\right.$ with $\beta \in \mathbb{R}^{d}, \gamma \in \mathbb{R}^{N}$ ) if there exists $\epsilon>0$ so that $L_{K}\left(\zeta_{0}+\beta \otimes B_{\epsilon}, \lambda\right)$ is bounded, where

$$
\zeta_{0}+\beta \otimes B_{\epsilon}:=\left\{\zeta \in \mathbb{R}^{d \times N}: \zeta=\zeta_{0}+\beta \otimes c \text { with }|c|<\epsilon\right\} .
$$

iii) Let $\zeta_{0, \alpha} \in \mathbb{R}^{d \times(N-1)}$ such that $\left(\zeta_{0, \alpha} \mid \nu_{0}\right) \in K$ for some $\nu_{0} \in \mathbb{R}^{d}$. We say that $K$ is uniformly stably bounded at $\zeta_{0, \alpha}$ in a direction $\lambda=\beta \otimes \gamma_{\alpha}$ with $\beta \in \mathbb{R}^{d}$ and $\gamma_{\alpha} \in \mathbb{R}^{N-1}$, if there exists $\epsilon>0$ such that for all $\nu \in \mathbb{R}^{d}$ with $\left(\zeta_{0, \alpha} \mid \nu\right) \in K$ the sets

$$
L_{K}\left(\left(\zeta_{0, \alpha}+\beta \otimes \bar{B}_{\epsilon}^{\prime} \mid \nu\right), \lambda\right)
$$

are bounded. Moreover, the bound is uniform for such $\nu \in \mathbb{R}^{d}$.

Finally we define:

Definition 3.0.5. Let $K_{1} \subset \mathbb{R}^{d \times N}$ be open, $\zeta_{0} \in K_{1}$ and $\lambda \in \mathbb{R}^{d \times N}$. We say that $\zeta_{0}$ is stably contained in $K_{1}$ in the direction $\lambda$ if there exists $\epsilon>0$ so that $L_{K}\left(\zeta_{0}+\beta \otimes \bar{B}_{\epsilon}, \lambda\right) \subset K_{1}$.

The following result which can be found in [14] says, roughly speaking, that if $K$ is bounded at $\zeta_{0}$ in a rank one direction $\lambda$ and this boundedness (in the same direction) is preserved under small perturbations of $\zeta_{0}$ along rank-one compatible directions, then we can ensure existence of solutions of $(P)$. Our primary goal will be to ensure that, under appropriate conditions on $f$ and $K, \bar{f}$ and $\bar{K}$ satisfy its hypothesis. 
Corollary 3.0.1. Let $\Omega \subset \mathbb{R}^{N}$ be a bounded open set, $f: \mathbb{R}^{d \times N} \rightarrow \mathbb{R}$ a lower semicontinuous function and let $\zeta_{0} \in K$ where

$$
K=\left\{\zeta \in \mathbb{R}^{d \times N}: Q_{N} f(\zeta)<f(\zeta)\right\} .
$$

If there exists a rank-one direction $\lambda \in \mathbb{R}^{d \times N}$ such that

i) $K$ is stably bounded at $\zeta_{0}$ in the direction $\lambda=\beta \otimes \gamma$,

ii) $Q_{N} f$ is quasiaffine on the set $L_{K}\left(\zeta_{0}+\beta \otimes \bar{B}_{\epsilon}, \lambda\right)$,

then the problem

$$
(P) \quad \inf \left\{\int_{\Omega} f(\nabla u(x)) d x: u \in u_{\zeta_{0}}+W_{0}^{1, \infty}\left(\Omega ; \mathbb{R}^{d}\right)\right\}
$$

has a solution $\bar{u} \in u_{\zeta_{0}}+W_{0}^{1, \infty}\left(\Omega ; \mathbb{R}^{d}\right)$.

We will also need the following definition:

Definition 3.0.6. (Uniform quasiaffinity in a direction $\lambda$ ). Let $f: \mathbb{R}^{d \times N} \rightarrow$ $\mathbb{R}$ and $K=\left\{Q_{N} f<f\right\}$.

We say that $Q_{N} f$ is uniformly quasiaffine at $\zeta_{0, \alpha} \in \mathbb{R}^{d \times(N-1)}$ in a direction $\lambda=\beta \otimes \gamma_{\alpha}$ with $\beta \in \mathbb{R}^{d}$ and $\gamma_{\alpha} \in \mathbb{R}^{N-1}$, if

a) there exists $\nu_{0} \in \mathbb{R}^{d}$ such that $\left(\zeta_{0, \alpha} \mid \nu_{0}\right) \in K$

b) there exists $\epsilon>0$ such that $Q_{N} f$ is quasiaffine on the set

$$
\bigcup_{\left\{\nu \in \mathbb{R}^{d}:\left(\zeta_{0, \alpha} \mid \nu\right) \in K\right\}} L_{K}\left(\left(\zeta_{0, \alpha}+\beta \otimes \bar{B}_{\epsilon}^{\prime} \mid \nu\right), \lambda\right) .
$$

\section{Dimension reduction}

Dimension reduction techniques, where mechanical properties of lower dimensional domains are derived via a $\Gamma$-convergence (cf. [1], [11], [15]) limiting procedure of variational problems in domains with vanishing thickness, have used extensively following the work of Le Dret and Raoult (cf. $[22])$.

We recall in this section the dimension reduction results of Le Dret and Raoult (cf. [22]). Note that this results, in the context of three-dimensional elasticity are stated for $N=d=3$ but it is a simple exercise to check that they hold for any $N \geq 2, d \geq 1$. Let $f: \mathbb{R}^{d \times N} \rightarrow \mathbb{R}$ a continuous function satisfying in addition:

$\left(H_{p}\right)$ : there exists $C>0$ such that

$$
\frac{1}{C}\left(|A|^{p}\right)-C \leq f(A) \leq C\left(1+|A|^{p}\right)
$$


for all $A \in \mathbb{R}^{d \times N}$ and for some $1<p<\infty$;

For $w \subset \mathbb{R}^{N-1}$ open bounded with Lipschitz boundary let

$$
\left.\Omega_{\epsilon}=w \times\right]-\frac{\epsilon}{2}, \frac{\epsilon}{2}[
$$

where $\epsilon \in] 0,1]$.

We consider the problems:

$$
\inf \left\{\int_{\Omega_{\epsilon}} f(\nabla v) d x, v \in W^{1, p}\left(\Omega_{\epsilon} ; \mathbb{R}^{d}\right)\right\}
$$

Changing variables i.e. setting $u\left(x_{\alpha} \mid x_{N}\right)=v\left(x_{\alpha} \mid \epsilon x_{N}\right)$, the rescaled energy (i.e. divided by $\epsilon$ ) is

$$
\int_{\Omega} f\left(\nabla_{\alpha} u \mid \frac{\nabla_{N} u}{\epsilon}\right) d x
$$

and the scaled problem $\left(P_{\epsilon}\right)$ is written as

$$
\inf \left\{\int_{\Omega} f\left(\nabla_{\alpha} u \mid \frac{\nabla_{N} u}{\epsilon}\right) d x, u \in W^{1, p}\left(\Omega ; \mathbb{R}^{d}\right)\right\} .
$$

Taking into account that we want to consider as targets functions $u \in$ $u_{\zeta_{\alpha}}+W_{0}^{1, p}\left(w ; \mathbb{R}^{d}\right)$ we define:

$$
\begin{array}{r}
I(u)=\inf _{u_{n}}\left\{\liminf _{\epsilon_{n} \rightarrow 0} \int_{\Omega} f\left(\nabla_{\alpha} u_{n} \mid \frac{\nabla_{N} u_{n}}{\epsilon_{n}}\right) d x,\left.u_{n} \in W^{1, p}\left(\Omega ; \mathbb{R}^{d}\right) u_{n}\right|_{\Gamma}=u\right. \\
\left.u_{n} \underset{n \rightarrow \infty}{\stackrel{L^{p}}{\rightarrow}} u\right\}
\end{array}
$$

Using $\Gamma$-convergence techniques it was shown by Le Dret and Raoult for $N=d=3$ (cf. [22]) (se also [4], [5], [6], [7] and [19]) that $I($.$) ( under the$ hypotheses $\left(H_{p}\right)$ on $\left.f\right)$ admits the following integral representation:

Theorem 4.0.1. Let $f: \mathbb{R}^{d \times N} \rightarrow \mathbb{R}$ continuous and satisfying $\left(H_{p}\right)$ for some $1<p<\infty$. For $\zeta_{\alpha} \in \mathbb{R}^{d \times(N-1)}$ let $u \in u_{\zeta_{\alpha}}+W_{0}^{1, p}\left(w ; \mathbb{R}^{d}\right)$. Then

$$
\begin{aligned}
I(u)= & \inf _{u_{n}}\left\{\liminf _{\epsilon_{n} \rightarrow 0} \int_{\Omega} f\left(\nabla_{\alpha} u_{n} \mid \frac{\nabla_{N} u_{n}}{\epsilon_{n}}\right) d x, u_{n} \in W^{1, p}\left(\Omega ; \mathbb{R}^{d}\right)\right. \\
& \left.\left.u_{n}\right|_{\Gamma}=u, u_{n} \underset{n \rightarrow \infty}{\stackrel{L^{p}}{\rightarrow}} u\right\} \\
= & \int_{w} Q_{N-1} \bar{f}\left(\nabla u\left(x_{\alpha}\right)\right) d x_{\alpha} .
\end{aligned}
$$


It is a simple exercise to check that the same representation holds for any $N>1, d \geq 1$.

\section{Sufficient conditions for existence of solutions}

5.1. Sufficient conditions for existence of solutions. Motivated by the result reviewed in the previous section we aim to derive conditions on $f$ and $K$ that are sufficient to ensure that the following problem admits solution:

$$
(\bar{P}) \quad \inf \left\{\int_{w} \bar{f}(\nabla v(x)) d x, v \in v_{\zeta_{0, \alpha}}+W_{0}^{1, \infty}\left(w ; \mathbb{R}^{d}\right)\right\},
$$

where $\zeta_{0, \alpha} \in \bar{K}:=\left\{\zeta_{\alpha}: Q_{N-1} \bar{f}\left(\zeta_{\alpha}\right)<\bar{f}\left(\zeta_{\alpha}\right)\right\}$.

However, as it will be clear latter on, in general we have to restrict $\zeta_{\alpha}$ to a subset of $\bar{K}$ where we can ensure quasiaffinity conditions on $\bar{f}$.

We start with the following couple of Lemmas:

Lemma 5.1. Let $f: \mathbb{R}^{d \times N} \rightarrow \mathbb{R}$ and $\zeta_{\alpha} \in \mathbb{R}^{d \times(N-1)}$. Then

$$
Q_{N}^{-} f\left(\zeta_{\alpha}\right) \geq Q_{N-1} \bar{f}\left(\zeta_{\alpha}\right)
$$

Proof. By definition, for all $\zeta \in \mathbb{R}^{d \times N}, \zeta=\left(\zeta_{\alpha} \mid \zeta_{N}\right)$,

$$
\begin{array}{r}
Q_{N} f(\zeta)=\inf \left\{\int_{Q} f(\zeta+\nabla v(x)) d x, v \in W_{0}^{1, \infty}\left(Q ; \mathbb{R}^{d}\right)\right\} \\
=\inf \left\{\int_{Q} f\left(\zeta_{\alpha}+\nabla_{\alpha} v(x) \mid \zeta_{N}+\nabla_{N} v(x)\right) d x, v \in W_{0}^{1, \infty}\left(Q ; \mathbb{R}^{d}\right)\right\} \\
\geq \inf \left\{\int_{Q} \bar{f}\left(\zeta_{\alpha}+\nabla_{\alpha} v(x)\right) d x, v \in W_{0}^{1, \infty}\left(Q ; \mathbb{R}^{d}\right)\right\} \\
=\inf \left\{\int_{I} \int_{Q^{\prime}} \bar{f}\left(\zeta_{\alpha}+\nabla v^{x_{N}}\left(x_{\alpha}\right)\right) d x_{\alpha} d x_{N}, v^{x_{N}} \in W_{0}^{1, \infty}\left(Q^{\prime} ; \mathbb{R}^{d}\right)\right\} \\
\geq \int_{I} \inf \left(\int_{Q^{\prime}} \bar{f}\left(\zeta_{\alpha}+\nabla w\left(x_{\alpha}\right)\right) d x_{\alpha}, w \in W_{0}^{1, \infty}\left(Q^{\prime} ; \mathbb{R}^{d}\right)\right) \\
\geq Q_{N-1} \bar{f}\left(\zeta_{\alpha}\right),
\end{array}
$$

where $v^{x_{N}}\left(x_{\alpha}\right):=v\left(x_{\alpha} \mid x_{N}\right)$. The arbitrariness of $\zeta_{N}$ leads to the desired result.

Lemma 5.2. Suppose that $f$ is continuous and satisfies $\left(H_{p}\right)$. Then, $\bar{f}$ is continuous. Moreover 


$$
\frac{1}{C}\left(\left|\zeta_{\alpha}\right|^{p}\right)-C \leq \bar{f}\left(\zeta_{\alpha}\right) \leq C\left(1+\left|\zeta_{\alpha}\right|^{p}\right)
$$

Proof. Let $\left(\zeta_{\alpha, n} \mid \nu_{n}\right) \rightarrow\left(\zeta_{\alpha} \mid \nu\right)$. Then clearly

$$
\limsup \bar{f}\left(\zeta_{\alpha, n}\right) \leq f\left(\zeta_{\alpha} \mid \nu\right),
$$

and from the arbitrariness of $\nu \in \mathbb{R}^{d}$ we conclude that $\bar{f}$ is upper semicontinuous. On the other hand, by definition of $\bar{f}$, we can pick $\nu_{n}$ such that

$$
f\left(\zeta_{\alpha, n} \mid \nu_{n}\right) \leq \bar{f}\left(\zeta_{\alpha, n}\right)+\frac{1}{n} .
$$

By $\left(H_{p}\right)$ we have that

$$
\frac{1}{C}\left(\left|\nu_{n}\right|^{p}\right)-C \leq f\left(\zeta_{\alpha, n} \mid \nu_{n}\right) \leq \bar{f}\left(\zeta_{\alpha, n}\right)+\frac{1}{n} \leq C\left(1+\left|\zeta_{\alpha, n}\right|^{p}\right)+\frac{1}{n}
$$

and we conclude that (up to a subsequence), $\nu_{n} \rightarrow \nu$. Therefore

$$
\bar{f}\left(\zeta_{\alpha}\right) \leq f\left(\zeta_{\alpha} \mid \nu\right) \leq \liminf _{n \rightarrow \infty} f\left(\zeta_{\alpha, n} \mid \nu_{n}\right) \leq \liminf _{n \rightarrow \infty} \bar{f}\left(\zeta_{\alpha, n}\right) .
$$

Note that the coercivity hypothesis on $f$ implies that in fact

$$
\bar{f}\left(\zeta_{\alpha}\right)=f\left(\zeta_{\alpha} \mid \nu_{\zeta_{\alpha}}\right)
$$

for some $\nu_{\zeta_{\alpha}} \in \mathbb{R}^{d}$. Hence

$$
\bar{f}\left(\zeta_{\alpha}\right)=f\left(\zeta_{\alpha} \mid \nu_{\zeta_{\alpha}}\right) \geq \frac{1}{C}\left(\left|\zeta_{\alpha}\right|^{p}\right)-C .
$$

Therefore $\bar{f}$ is nonnegative outside a compact set $C$. Since $|\bar{f}|$ is continuous it is bounded on $C$ and hence, for $\zeta_{\alpha} \notin C$,

$$
\left|\bar{f}\left(\zeta_{\alpha}\right)\right|=\bar{f}\left(\zeta_{\alpha}\right) \leq f\left(\zeta_{\alpha} \mid 0\right) \leq C\left(1+\left|\left(\zeta_{\alpha} \mid 0\right)\right|^{p}\right)=C\left(1+\left|\zeta_{\alpha}\right|^{p}\right) .
$$

Since trivially

$$
Q_{N-1}\left(\overline{Q_{N}} f\left(\zeta_{\alpha}\right)\right) \leq Q_{N-1} \bar{f}\left(\zeta_{\alpha}\right)
$$

from Lemma 5.1 we conclude that in fact

$$
Q_{N-1}\left(\overline{Q_{N}} f\left(\zeta_{\alpha}\right)\right)=Q_{N-1} \bar{f}\left(\zeta_{\alpha}\right) .
$$

However in general $Q_{N}^{-} f$ is not quasiconvex.

For the sake of illustration we state here the following result (Corollary 11 in $[22])$ : 
Corollary 5.2.1. Let $f: \mathbb{R}^{3 \times 3} \rightarrow \mathbb{R}$ continuous and satisfying $\left(H_{p}\right)$. Suppose further that $f$ is frame-indifferent, i.e.

$$
\forall \zeta \in \mathbb{R}^{3 \times 3}, R \in S O(3), f(R \zeta)=f(\zeta)
$$

and that $f(I)=0, f(\zeta)>0$ if $\zeta \notin S O(3)$. Then $Q_{N-1} \bar{f}<\bar{f}$.

Hence, in general we could have that

$$
Q_{N-1} \bar{f}<\overline{Q_{N} f}
$$

and therefore are unable to derive quasiaffinity conditions on $Q_{N-1} \bar{f}$ from those assumed in $Q_{N} f$.

However, as was also pointed out by Le Dret and Raoult (cf. [22]), there are some situations were we have that $Q_{N-1} \bar{f}=\bar{Q}_{N} f$, namely:

i) in the scalar case; more precisely, if $W: \mathbb{R}^{d \times N} \rightarrow \mathbb{R}$ is convex then $\bar{W}$ is convex. Hence, if $Q_{N} f=C_{N} f$ ( in particular in the scalar case) we have that $\overline{Q_{N}} f$ is convex.

ii) in the case where $\bar{f}\left(\zeta_{\alpha}\right)=f\left(\zeta_{\alpha} \mid z\right)$, for $z \in \mathbb{R}^{d}$ independent of $\zeta_{\alpha}$.

(cf. the Remark following Corollary 11 and Proposition 12 in [22]).

It is easy to check that $\left\{\zeta_{\alpha}: Q_{N-1} \bar{f}\left(\zeta_{\alpha}\right)<\overline{Q_{N}} f\left(\zeta_{\alpha}\right)\right\} \subset \bar{K}$. In order to adress more general situations we define $\tilde{K}$ as the closure in $\mathbb{R}^{d \times(N-1)}$ of the set $\left\{\zeta_{\alpha}: Q_{N-1} \bar{f}\left(\zeta_{\alpha}\right)<\overline{Q_{N}} f\left(\zeta_{\alpha}\right)\right\}$ and we will therefore restrict ourselves to derive sufficient conditions for existence of solutions of $(\bar{P})$ for $\zeta_{\alpha} \in \bar{K}_{1}:=\bar{K} \backslash \tilde{K}$.

In Le Dret and Raoult (cf. [22]) one can find an explicit example where $\bar{K}_{1}$ is non-empty (for the case of the Sain-Venant-Kirchoff materials).

The following Proposition says, roughly speaking, that we can pass the quasiaffinity hypothesis on $Q_{N} f$ onto $Q_{N-1} \bar{f}$ as long as we stay in the "good" set $\bar{K}_{1}$.

Proposition 5.2.1. Let $f: \mathbb{R}^{d \times N} \rightarrow \mathbb{R}$ a continuous function satisfying $\left(H_{p}\right)$ and let $\zeta_{0, \alpha}$ be stably contained in $\bar{K}_{1}$ along a direction $\lambda=\beta \otimes \gamma_{\alpha} \in$ $R^{d \times(N-1)}$. Suppose further that $Q_{N}$ is uniformly quasiaffine at $\zeta_{0, \alpha}$ in the direction $\lambda$. Then $Q_{N-1} \bar{f}$ is quasiaffine on $L_{\bar{K}}\left(\zeta_{0, \alpha}+\beta \otimes \bar{B}_{\epsilon}^{\prime}, \lambda\right)$, for some $\epsilon>0$.

Proof. Since $Q_{N} f$ is uniformly quasiaffine at $\zeta_{0, \alpha}$ in a direction $\lambda=\beta \otimes \gamma_{\alpha} \in$ $R^{d \times(N-1)}$ there exists $\epsilon_{0}>0$ such that, for all $\nu \in \mathbb{R}^{d}$ with $\left(\zeta_{0, \alpha} \mid \nu\right) \in K$, we have that $Q_{N} f$ is quasiaffine on the set $L_{K}\left(\left(\zeta_{0, \alpha}+\beta \otimes \overline{B_{\epsilon_{0}}^{\prime}} \mid \nu\right), \beta \otimes \gamma\right)$. 
Since, by Lemma $5.2 \bar{f}$ is continuous and since $Q_{N-1} \bar{f}$ is lower semicontinuous (cf. [8]) we have that $\bar{K}$ is open and hence $\bar{K}_{1}$ is open. Therefore we can find $\epsilon_{1}>0$ such that $\zeta_{0, \alpha}+\beta \otimes \overline{B_{\epsilon_{1}}^{\prime}} \subset \overline{K_{1}}$. Moreover, since $\zeta_{0, \alpha}$ is stably contained in $\bar{K}_{1}$ along the direction $\lambda=\beta \otimes \gamma_{\alpha} \in R^{d \times(N-1)}$ there exists $\epsilon_{2}<\epsilon_{1}$ such that $L_{\bar{K}}\left(\zeta_{0, \alpha}+\beta \otimes \overline{B_{\epsilon_{2}}^{\prime}}, \beta \otimes \gamma\right) \subset \bar{K}_{1}$. Let now $\epsilon=\min \left\{\epsilon_{0}, \epsilon_{2}\right\}$ and consider $\zeta_{\alpha}^{1}, \zeta_{\alpha}^{2} \in L_{\bar{K}}\left(\zeta_{0, \alpha}+\beta \otimes \bar{B}_{\epsilon}^{\prime}, \lambda\right)$, more exactly satisfying $\zeta_{\alpha}^{i}=\zeta_{0, \alpha}+\beta \otimes c+t^{i} \lambda, i=1,2$ for some $c \in \mathbb{R}^{N-1},|c| \leq \epsilon$. Trivially for $\theta \in[0,1]$,

$$
Q_{N-1} \bar{f}\left(\theta \zeta_{\alpha}^{1}+(1-\theta) \zeta_{\alpha}^{2}\right) \leq \theta Q_{N-1} \bar{f}\left(\zeta_{\alpha}^{1}\right)+(1-\theta) Q_{N-1} \bar{f}\left(\zeta_{\alpha}^{2}\right) .
$$

Moreover since $\zeta_{\alpha}$ is stably contained in $\bar{K}_{1}$ along the direction $\lambda$,

$$
Q_{N-1} \bar{f}\left(\theta \zeta_{\alpha}^{1}+(1-\theta) \zeta_{\alpha}^{2}\right)=Q_{N}^{-} f\left(\theta \zeta_{\alpha}^{1}+(1-\theta) \zeta_{\alpha}^{2}\right)
$$

and we have that

$$
Q_{N-1} \bar{f}\left(\theta \zeta_{\alpha}^{1}+(1-\theta) \zeta_{\alpha}^{2}\right)=Q_{N} f\left(\theta \zeta_{\alpha}^{1}+(1-\theta) \zeta_{\alpha}^{2} \mid \nu_{0}\right)
$$

for some $\nu_{0} \in \mathbb{R}^{d}$, since $Q_{N} f$ is continuous and satisfies naturally the same coercivity hypothesis than $f$.

Now it is clear that

$$
Q_{N} f\left(\theta \zeta_{\alpha}^{1}+(1-\theta) \zeta_{\alpha}^{2} \mid \nu_{0}\right)<f\left(\theta \zeta_{\alpha}^{1}+(1-\theta) \zeta_{\alpha}^{2} \mid \nu_{0}\right)
$$

otherwise we would have that

$$
Q_{N-1} \bar{f}\left(\theta \zeta_{\alpha}^{1}+(1-\theta) \zeta_{\alpha}^{2}\right)=\bar{f}\left(\theta \zeta_{\alpha}^{1}+(1-\theta) \zeta_{\alpha}^{2}\right)
$$

and this would contradict our choice of $\zeta_{\alpha}^{1}, \zeta_{\alpha}^{2}$. From (5.6) and our choice of $\epsilon$ we have that

$$
\left(\theta \zeta_{\alpha}^{1}+(1-\theta) \zeta_{\alpha}^{2} \mid \nu_{0}\right) \in L_{K}\left(\left(\zeta_{0, \alpha}+\beta \otimes \overline{B_{\epsilon_{0}}^{\prime}} \mid \nu_{0}\right), \lambda\right)
$$

and from the arbitrariness of $\theta$ we also have

$$
\left(\zeta_{\alpha}^{i} \mid \nu_{0}\right) \in L_{K}\left(\left(\zeta_{0, \alpha}+\beta \otimes \overline{B_{\epsilon_{0}}^{\prime}} \mid \nu_{0}\right), \lambda\right), i=1,2 .
$$

Since $Q_{N} f$ is quasiaffine on $L_{K}\left(\left(\zeta_{0, \alpha}+\beta \otimes \overline{B_{\epsilon_{0}}^{\prime}} \mid \nu_{0}\right), \lambda\right)$ and from our choice of $\zeta_{\alpha}^{i}, i=1,2$,

$$
\begin{array}{r}
Q_{N-1} \bar{f}\left(\theta \zeta_{\alpha}^{1}+(1-\theta) \zeta_{\alpha}^{2}\right)=Q_{N} f\left(\theta \zeta_{\alpha}^{1}+(1-\theta) \zeta_{\alpha}^{2} \mid \nu_{0}\right) \\
=\theta Q_{N} f\left(\zeta_{\alpha}^{1} \mid \nu_{0}\right)+(1-\theta) Q_{N} f\left(\zeta_{\alpha}^{2} \mid \nu_{0}\right) \\
\geq \theta Q_{N}^{-} f\left(\zeta_{\alpha}^{1}\right)+(1-\theta) Q_{N}^{-} f\left(\zeta_{\alpha}^{2}\right) \\
=\theta Q_{N-1} \bar{f}\left(\zeta_{\alpha}^{1}\right)+(1-\theta) Q_{N-1} \bar{f}\left(\zeta_{\alpha}^{2}\right)
\end{array}
$$


The equivalence between quasiaffinity and rank one affinity (cf. [16]) concludes the proof of the claim.

We are now in position to state the main result:

Theorem 5.2.1. Let $f: \mathbb{R}^{d \times N} \rightarrow \mathbb{R}$ continuous and satisfying $\left(H_{p}\right)$. Let $\zeta_{0, \alpha} \in \mathbb{R}^{d \times(N-1)}$ be stably contained in $\bar{K}_{1}$ along a direction $\lambda=\beta \otimes \gamma_{\alpha} \in$ $R^{d \times N-1}$. Suppose further that $Q_{N} f$ is uniformly quasiaffine at $\zeta_{0, \alpha}$ in the direction $\lambda$ and that $K$ is uniformly stably bounded at $\zeta_{0, \alpha}$ in the direction $\lambda$. Then

$$
(\bar{P}) \quad \inf \left\{\int_{w} \bar{f}(\nabla v(x)) d x, v \in v_{\zeta_{0, \alpha}}+W_{0}^{1, \infty}\left(w ; \mathbb{R}^{d}\right)\right\}
$$

attains solution.

Proof. We start by noting that if $\zeta_{\alpha}$ is stably contained in $\bar{K}_{1}$ there exist $\nu_{\alpha}^{1}, \nu_{\alpha}^{2} \in \mathbb{R}^{d}$ such that

$$
Q_{N-1} \bar{f}\left(\zeta_{\alpha}\right)=Q_{N} f\left(\zeta_{\alpha} \mid \nu_{\zeta_{\alpha}}^{1}\right), \quad \bar{f}\left(\zeta_{\alpha}\right)=f\left(\zeta_{\alpha} \mid \nu_{\alpha}^{2}\right)
$$

and

$$
Q_{N} f\left(\zeta_{\alpha} \mid \nu_{\zeta_{\alpha}}^{1}\right)<f\left(\zeta_{\alpha} \mid \nu_{\alpha}^{2}\right)
$$

Moreover $\nu_{\zeta_{\alpha}}^{1}$ can be chosen so that

$$
\left(\zeta_{\alpha} \mid \nu_{\zeta_{\alpha}}^{1}\right) \in K
$$

In fact, suppose that for all $\nu \in \mathbb{R}^{d}$ such that $Q_{N-1} \bar{f}\left(\zeta_{\alpha}\right)=Q_{N} f\left(\zeta_{\alpha} \mid \nu\right)$ we have that $\left(\zeta_{\alpha} \mid \nu\right) \notin K$. Then trivially, $Q_{N-1} \bar{f}\left(\zeta_{\alpha}\right)=Q_{N} f\left(\zeta_{\alpha} \mid \nu\right)=f\left(\zeta_{\alpha} \mid \nu\right) \geq$ $\bar{f}\left(\zeta_{\alpha}\right)$, a contradiction.

Let $\epsilon$ is the minimum from those taken from the hypothesis of uniform stable boundedness of $K$ at $\zeta_{0, \alpha}$ along the direction $\lambda$ and from the hypothesis of $\zeta_{0, \alpha}$ being stably contained in $\bar{K}_{1}$. Now given any $c \in \mathbb{R}^{N}$ such that $|c|<\epsilon$ it is clear, from the hypothesis of uniform stable boundedness, that for any $\nu$ such that $\left(\zeta_{\alpha} \mid \nu\right) \in K$, we have that

$$
\left(\zeta_{\alpha} \mid \nu\right)+\beta \otimes c+t(\lambda \mid 0) \notin K
$$

as long as we take $|t|$ large enough. From the note at the beginning of the proof, since $\zeta_{0, \alpha}$ is stably contained in $\bar{K}_{1}$, we conclude that for $c^{\prime} \in \mathbb{R}^{N-1}$ with $\left|c^{\prime}\right|<\epsilon$,

$$
\zeta_{\alpha}+\beta \otimes c^{\prime}+t \lambda \notin \bar{K}
$$

In other words, $\bar{K}$ is stably bounded at $\zeta_{0, \alpha}$ along the direction $\lambda$. The result now follows immediately from Proposition 5.2.1 and Corollary 3.0.1. 
In the case $d=1$, taking into consideration the previous comments, Theorem 5.2.1 reads simply:

Theorem 5.2.2. Let $N>2$ and $f: \mathbb{R}^{N} \rightarrow \mathbb{R}$ continuous and satisfying $\left(H_{p}\right)$. Let $\zeta_{0, \alpha} \in \bar{K}$. Suppose further that $C_{N} f$ is uniformly affine at $\zeta_{0, \alpha}$ in a direction $\lambda=\left(\lambda_{\alpha} \mid 0\right)$ and that $K$ is uniformly stably bounded at $\zeta_{0, \alpha}$ in the direction $\lambda$. Then

$$
(\bar{P}) \quad \inf \left\{\int_{w} \bar{f}(\nabla v(x)) d x, v \in v_{\zeta_{0, \alpha}}+W_{0}^{1, \infty}(w)\right\}
$$

attains solution.

Remark 5.2.1. From the proof of Theorem 5.2.1 it is clear that we can weaken the hypothesis of uniform stable boundedness, namely by dropping the uniformity of the bound on $\nu$, as long as we impose in addition that the set $\left\{\left(\zeta_{\alpha} \mid t\right), t \in \mathbb{R}\right\} \cap K$ is bounded.

Remark 5.2.2. If $d=1$ and in addition $N=2$, it is clear that we can drop the uniform affinity hypothesis since $Q_{1} \bar{f}$ will always be affine at $\zeta_{0, \alpha} \in \bar{K}$. On this particular case we just need to impose the hypothesis of uniform stable boundedness at $\zeta_{0, \alpha}$ in the direction $e_{1}$.

5.2. Examples. The following trivial example shows that in general $\bar{f}$ is not convex.

\section{Example 1:}

Let $g: \mathbb{R} \rightarrow \mathbb{R}$ non-convex and set $f: \mathbb{R}^{2} \rightarrow \mathbb{R}$ such that:

$$
f(x \mid 0)=g(x), f(x \mid t)=f(x \mid-t) \geq f(x \mid 0) .
$$

Then, clearly $\bar{f}=g$ is non-convex.

Note also that, even if $\left(\zeta_{\alpha} \mid t\right) \in K$ for all $t \in \mathbb{R}$, we cannot conclude that $\bar{f}\left(\zeta_{\alpha}\right)>\bar{C} f\left(\zeta_{\alpha}\right)$, i.e. that $\zeta_{\alpha} \in \bar{K}$. In fact:

Example 2: Consider $g: \mathbb{R} \rightarrow \mathbb{R}^{+}$non-convex and bounded and set $f: \mathbb{R}^{2} \rightarrow \mathbb{R}$ such that:

$$
f(x \mid 0)=g(x), f(x \mid t)=f(x \mid-t)=\frac{1}{|1-| t||} g(x) .
$$

Then, clearly we could have $K$ unbounded at some $x_{1}$ in the direction $e_{2}$ and $\bar{f}\left(x_{1}\right)=\bar{C} f\left(x_{1}\right)=0$.

If we assume that $K$ is bounded on the direction $e_{N}$ at $\zeta_{\alpha}$ (meaning that for all $t \in \mathbb{R}$ such that $\left(\zeta_{\alpha} \mid t\right) \in K$ we have that $L_{K}\left(\left(\zeta_{\alpha} \mid t\right), e_{N}\right)$ is bounded) 
and that $C f$ is affine on $K$ leads clearly to $\overline{C f}\left(\zeta_{\alpha}\right)=C f\left(\zeta_{0} \mid t_{0}\right)=f\left(\zeta_{0} \mid t_{0}\right)$, for some $t_{0} \in \mathbb{R}$ since the infimum is attained at the boundary. Hence $\bar{f}\left(\zeta_{\alpha}\right)=\bar{C} f\left(\zeta_{\alpha}\right)$, and Problem $(\bar{P})$ has the trivial solution $v=v_{\zeta_{\alpha}}$.

Following Example 2 we could construct a function $f: \mathbb{R}^{2} \rightarrow \mathbb{R}$ such that $K$ is bounded on the direction $e_{2}$, affine on the direction $e_{1}$ but with $\bar{C} f(x)=C f\left(x \mid t_{0}\right)<f\left(x \mid t_{0}\right)$ for some $t_{0} \in \mathbb{R}$.

\section{Acknowledgements:}

The author would like to thank Prof. Bernard Dacorogna for his valuable comments on earlier drafts of this paper.

This work was partially supported by the Fundação para a Ciência e Tecnologia (FCT- Portugal).

\section{References}

[1] De Giorgi, E. and L. Ambrosio, Un nuovo tipo di funzionale dell calculo delle variazioni, Atti. Acad. Naz. Lincei Rend. Cl. Sci. Fis. Mat. Natur. 82 (1998), 199-210.

[2] Ambrosio, L. and G. Dal Maso, On the relaxation in $B V\left(\Omega ; \mathbb{R}^{m}\right)$ of Quasi-convex integrals, J. Funct. Anal. 109 (1992), 76-97.

[3] Braides, A., $\Gamma$-convergence for beginners, Oxford Lecture Series in Mathematics and its Applications, 22, Oxford University Press, Oxford, 2002.

[4] Bhattacharya, R., I. Fonseca and G. Francfort, An asymptotic study of the debonding of thin films, Rational Mech. Anal. 161 (2002), 205-229.

[5] Bhattacharya, R. and R. D. James, A theory of thin films of martensitic materials with applications to microactuators, J. Mech. Phys. Solids 47 (1999), 531-576.

[6] Braides, A., I. Fonseca and G. Francfort, 3D-2D asymptotic analysis for inhomogeneous thin films, Indiana Univ. Math. J. 49 (2000), 1367-1404.

[7] Braides, A. and I. Fonseca, Brittle Thin Films, Appl. Math. Optm. 44 (2001) 299-323.

[8] Barron, E.N., R. R. Jensen, C.Y. Wang, Lower semicontinuity of $L^{\infty}$ functionals, Ann. I. H. Poincaré- AN 184 (2001) 495-517.

[9] Bocea, M. and I. Fonseca, Equi-integrability results for $3 D-2 D$ dimension reduction problems, ESAIM: Control, Optimization and Calculus of Variations 7 (2002), 443-470.

[10] Dal Maso, G. An Introduction to $\Gamma$-convergence, Birkhäuser, 1993.

[11] De Giorgi, E. and G. Dal Maso, $\Gamma$-convergence and calculus of variations, Mathematical theories of optimization (Genova 1981), 121-143, Lecture Notes in Math. 979, Springer, Berlin, 1983.

[12] De Giorgi, E. and T. Franzoni, Su un tipo di convergenza variazionale, Atti Accad. Naz. Lincei Rend. Cl. Sci. Fis. Mat. Natur. (8) $\mathbf{5 8}$ (1975), No. 6, 842-850.

[13] De Giorgi, E. and G. Letta, Une solution générale de convergence faible pour des fonctions croissantes d'emsemble, Ann. Sc. Norm. Sup. Pisa Cl. Sci (4) 4 (1977), 61-99.

[14] Dacorogna, B. , G. Pisante and A.M. Ribeiro, On non quasiconvex problems in the calculus of variations, Discrete and Continuous Dynamical Systems (2005).

[15] Dal Maso, G., An introduction to $\Gamma$ - convergence, Progress in Nonlinear Differential Equations and their Applications, 8. Birkhäuser Boston, Inc., Boston, MA, 1993.

[16] Dacorogna, B., Direct methods in the calculus of variations, Applied Math. Sciences, 78, Springer, Berlin (1989).

[17] Dacorogna, B. and P. Marcellini, Existence of minimizers for non quasiconvex integrals, Arch. Rational Mech. Anal. 131 (1995), 359-399.

[18] Dacorogna, B. and P. Marcellini, Implicit partial differential equations, Birkhauser, Boston (1999). 
Sufficient conditions for existence of solutions of a lower dimensional variational problem $\mathbf{5 1}$

[19] Fonseca I. and G. Francfort, On the inadequacy of scaling of linear elasticity for 3D-2D asymptotics in a nonlinear setting, J. Math. Pures Appl. 80 (2001), 547-562.

[20] Fonseca, I. and G. Leoni, Modern Methods in the Calculus of Variations: : $L^{p}$ spaces, Springer, New York, 2007.

[21] Fonseca, I. and J. Malý, Relaxation of multiple integrals in sobolev spaces below the growth exponent for the energy density, Annal. Ins. J. Poincaré (C) Non linear Analysis 14 (1997), No. 3, 309-338.

[22] Le Dret, H. and A. Raoult, The nonlinear membrane model as variational limit of nonlinear three-dimensional elasticity, J. Math. Pures Appl. 74 (1995), 549-578.

São Paulo J.Math.Sci. 5, 1 (2011), 37-51 